\title{
INTELIGENCIA EMOCIONAL RASGO EN LA FORMACIÓN INICIAL DEL PROFESORADO
}

\author{
David Molero \\ Antonio Pantoja-Vallejo \\ Miriam Galiano-Carrión \\ Universidad de Jaén
}

\begin{abstract}
RESUMEN: Este estudio analiza la Inteligencia Emocional (IE) en docentes durante su formación inicial siguiendo el modelo IE rasgo, en concreto el modelo del bienestar de Bar-On (2002; 2006). Participaron 460 estudiantes (edad en años $M=22.57, \mathrm{DT}= \pm 3.39$ ) de la Universidad de Jaén (España) que respondieron a la escala EQ-i Short Form en su versión española (LópezZafra, Pulido-Martos y Berrios-Martos, 2014), que contempla 4 factores (Interpersonal, Adaptabilidad, Manejo del Estrés e Intrapersonal). Existen diferencias significativas $(p<.05)$ en diversos factores en función del género, edad, grado de los participantes y nivel educativo de los mismos. Las variables consideradas en el estudio de regresión que más predicen la IE global son Manejo del Estrés, seguido de la Adaptabilidad, Intrapersonal e Interpersonal. Los resultados obtenidos son concordantes con los obtenidos en otros estudios realizados en contextos similares.
\end{abstract}

PALABRAS CLAVE: Inteligencia emocional, emociones, rasgos de la personalidad, inteligencia emocional rasgo, formación profesorado.

\section{TRAIT EMOTIONAL INTELLIGENCE IN INITIAL TEACHER TRAINING}

ABSTRACT: This study analyzes the emotional intelligence (EI) in teachers during their initial training following the trait El model, namely the wellness model Bar-On (2002; 2006). 460 students participated (age in years $M=22.57$, $\mathrm{SD}= \pm 3.39$ ) of the University of Jaen (Spain) who responded to the scale EQ-i Short Form Spanish version (López-Zafra, Pulido-Martos, \& Berrios-Martos, 2014), that includes 4 factors (Interpersonal, Adaptability, Stress management and Intrapersonal). There are significant differences $(p<.05)$ on various factors based on gender, age, degree of participants and the educational level of the same. The variables considered in the regression analysis that most predict 
global IE are Stress Management, Adaptability followed, Intrapersonal and Interpersonal. The results are consistent with those obtained in other studies in similar contexts.

KEYWORDS: Emotional intelligence, emotions, personality traits, trait emotional intelligence, teacher training.

Recibido: 21/03/2016

Aceptado: 15/07/2016

Correspondencia: David Molero López-Barajas, Universidad de Jaén, Facultad de Humanidades y Ciencias de la Educación, Departamento de Pedagogía, Campus "Las Lagunillas", s/n. C.P. 23071 Jaén (España). Email: dmolero@ujaen.es.

\section{INTRODUCCIÓN}

A partir de la publicación de Salovey y Mayer (1990) del primer artículo científico basado en la Inteligencia Emocional (a partir de ahora IE) se han desarrollado numerosas investigaciones en torno a este temática siendo un campo de estudio de creciente interés por la comunidad científica. La IE constituye el desarrollo psicológico más reciente en el campo de las emociones (Jiménez y López-Zafra, 2009; Salovey y Grewal, 2005), siendo considerada por algunos autores de contextos similares al nuestro como una interacción adecuada entre las emociones y los aspectos cognitivos, la cual permite al individuo un funcionamiento adaptado a su medio (Cazalla-Luna y Molero, 2016).

En torno al constructo IE se han creado dos modelos claramente diferenciados. El primero es el modelo de habilidad (Mayer y Salovey, 1997) que considera la IE como una capacidad de razonar en torno a las emociones y emplear este conocimiento emocional para mejorar y conducir el pensamiento, algo que permite resolver problemas sociales y adaptarse al medio ambiente (López-Zafra, Pulido-Martos y Berrios-Martos, 2014). Este primer modelo contempla que la IE es la capacidad de identificar, evaluar y diferenciar las emociones personales y grupales, saber usarlas par tomar decisiones, comprenderlas y regular tanto las emociones positivas como las negativas en uno mismo y en los otros (Mayer, Roberts y Barsade, 2008). La segunda concepción de la IE está basada en el modelo IE rasgo (Bar-On, 1997; Petrides y Furham, 2000, 2001), que considera que la IE es un conjunto de rasgos estables de la personalidad, competencias socioemocionales, motivaciones y habilidades cognitivas (Bar-On, 2006). Pero más allá de qué modelo teórico que se emplee para estudiar e investigar la IE, coincidimos con Rey y Extremera (2012) al considerar que el estudio de la IE abre una nueva visión en el análisis de las emociones en la que éstas pasan de ser elementos de los procesos cognitivos a fenómenos vitales del ser humano al ofrecer información útil para solucionar los problemas cotidianos.

La IE, tradicionalmente, ha sido evaluada a través de tres vías: medidas de autoinforme, medidas de ejecución e informes de evaluación $360^{\circ}$ por medio de observadores externos (Extremera, Fernández-Berrocal, Mestre y Guil, 2004; López-Zafra, Pulido-Martos, Berrios-Martos y Augusto-Landa, 2012; Roberts, Mac- 
Cann, Matthews y Zeidner, 2010). Si nos centramos en las medidas de autoinforme (auto-observación que el sujeto realiza de su propia conducta) se pueden diferenciar entre las que se basan en el modelo de habilidad y las que se basan en el modelo de rasgo. En este estudio no basamos en un instrumento de medida basado en el segundo modelo (rasgo), siguiendo el modelo de bienestar de Bar-On (2002; 2006) quien define la IE como un conjunto de competencias y destrezas personales, emocionales y sociales que favorecen las estrategias de afrontamiento y condicionan nuestra eficacia para comprender y relacionarlos con los demás, así como para resolver problemas del día a día. Esta propuesta considera que la IE está formada por 5 factores y 15 competencias que son: Intrapersonal (autoconciencia emocional, asertividad, independencia, autoconcepto y auto-actualización), Interpersonal (empatía, responsabilidad social y manejo de las relaciones interpersonales), Manejo del Estrés (tolerancia al estrés y control de impulsos), Estado de ánimo (búsqueda-mantenimiento de la felicidad y optimismo) y Adaptación (Estrategias de solución de problemas, prueba de realidad y flexibilidad). El modelo de bienestar puede evaluarse a través un instrumento, el EQ-i (Bar-On, 2002), del cual hay un versión breve EQ-i Short Form (Parker, Keefer y Wood, 2011), de la que se han realizado diversas adaptaciones al español en su versión para escolares jóvenes (Sáinz, Ferrándiz, Fernández y Ferrando, 2014), adolescentes (Ferrándiz, Hernández, Bermejo, Ferrando y Sáinz, 2012) o para población universitaria (López-Zafra et al., 2014).

Los resultados de los estudios previos indican que la IE rasgo correlaciona positivamente con una imagen corporal saludable (Swami, Begum y Petrides, 2010) y con enfoques emocionales correctos en cuidadores sanitarios (Weaving, Orgeta, Orrel y Petrides, 2014). La IE rasgo también correlaciona positivamente con la empatía (Marjanović y Dimitrijevic, 2014), consonancia emocional (Mikolajczak, Menil y Luminet, 2007), afectos positivos (Andrei y Petrides, 2013; Cazalla-Luna y Molero, 2014), la felicidad (Furnham y Christoforou, 2007), satisfacción con la vida (Adina y Clipa, 2012; Freudenthaler, Neubauer, Gabler, Scherl y Rindermann, 2008; Kong, Zhao y You, 2012), optimismo (Mikolajczak, Luminet, Leroy y Roy, 2007) y la comunicación (Jacobs, Sim y Zimmermann, 2015). En relación a las variables sociodemográficas consideradas en nuestro estudio, también hay evidencias de relaciones significativas entre la IE rasgo y género normalmente a favor de las mujeres (Fortes-Vilaltella, Oriol, Filella-Guiu, Del Arco y Soldevila, 2013; Marjanović y Dimitrijević, 2014; ValadezSierra, Borges del Rosal, Ruvalcaba-Romero, Villegas y Lorenzo, 2013); la edad a favor de los más mayores (Marjanović y Dimitrijević, 2014; Yeung, Wong y Lok, 2011), y el nivel educativo (Manoharan, 2008). Se ha demostrado validez incremental de la IE rasgo en varios aspectos de las dimensiones básicas de personalidad (Andrei, Smith, Surcinelli, Baldaro y Saklofske, 2015; Marjanović y Dimitrijević, 2014), afectos positivos y negativos (Andrei y Petrides, 2013) y la empatía (Marjanović y Dimitrijević, 2014).

Actualmente, una de las líneas de investigación que más interés genera dentro del campo de la IE es el análisis del papel que juegan las emociones en el contexto educativo y, sobre todo, profundizar en la influencia de la variable IE a la hora de determinar tanto el éxito académico de los estudiantes como su adaptación escolar (Sáinz et al., 2014). 
Los objetivos considerados en el estudio con carácter general son: (a) Analizar las puntuaciones de IE en los futuros docentes de Educación Infantil y Educación Primaria de la muestra (b) Estudiar la existencia de correlaciones significativas entre las dimensiones del instrumento considerado para evaluar la IE, EQi-C, adaptado al español por López-Zafra et al. (2014): Interpersonal, Adaptabilidad, Manejo del estrés e Intrapersonal, (c) Analizar la existencia de diferencias significativas en las puntuaciones de IE en función de las variables consideradas (género, edad, titulación y curso) y (d) Predecir qué variables de las consideradas en el estudio son las que más explican la valoración global de la IE.

\section{Método}

El trabajo que presentamos es de tipo no experimental empleando la estrategia de investigación asociativa, combinando los estudios comparativos y de predicción, realizándose medidas de carácter transversal (Ato, López y Benavente, 2013).

\section{Participantes}

Los participantes son estudiantes de los Grados de Magisterio Educación Infantil y Magisterio Educación Primaria de la Universidad de Jaén (España). Empleamos un muestreo no probabilístico de tipo accidental. La muestra está formada por un total de 460 estudiantes $(n=460)$ de los cuales 104 son hombres $(22.6 \%)$ y 356 son mujeres $(77.4 \%)$, ya que el contexto de estos grados la mayoría del alumnado son mujeres, siendo está proporción de género similar en el total de los matriculados en ambos grados en la Universidad de Jaén. La edad media (expresada en años) es de 22.57 \pm 3.39 ; 404 de los estudiantes son menores de 25 años (87.8\%) y 56 de ellos tienen 25 o más años (12.2\%). Participan 235 estudiantes del Grado de Magisterio de Educación Infantil (51.1\%) y 225 del Grado de Magisterio de Educación Primaria (48.9\%). Finalmente, en relación al nivel o curso, 200 son estudiantes de $3^{\circ}(43.5 \%)$ y 260 de $4^{\circ}(56.5 \%)$. Como puede apreciarse en todos los datos ofrecidos con anterioridad, se guarda una proporcionalidad entre el número de participantes en función del grado y del nivel (curso), mientras que son mayoría las mujeres y los menores de 25 años.

\section{Instrumentos}

\section{Cuestionario sociodemográfico}

Con el propósito de recabar información relevante se han incluido cuatro variables sociodemográficas: género (hombres vs. mujeres), edad ( $<25$ años vs. $\geq 25$ años), grado (Infantil vs. Primaria) y curso/nivel ( $3^{\circ}$ vs. $\left.4^{\circ}\right)$, con la intención de analizar la existencia de diferencias significativas en función de las mismas.

\section{EQ-i (Short Form) adaptación al español}

Este instrumento es una prueba de autoinforme, basada en el modelo de IE de Bar-On (2000; 2006) y en el instrumento de medida de la IE diseñado por este mismo autor, el 
Emotional Quotient Inventory (EQ-i). López-Zafra et al. (2014) validaron y adaptaron el mismo al español elaborando una versión corta (short form) compuesta por 28 ítems de una escala Likert de 5 puntos y 4 factores: Factor 1: Interpersonal (7 ítems), Factor 2: Adaptabilidad (5 ítems), Factor 3: Manejo del estrés (8 ítems) y Factor 4: Intrapersonal (8 ítems). Todas las cuestiones contempladas en el Factor 3 y Factor 4 al corregir el instrumento tienen que recodificarse (puntuaciones inversas). En nuestra muestra la fiabilidad de las puntuaciones (consistencia interna informada) para cada factor es de: .67 para Interpersonal, .74 en Adaptabilidad, .88 Manejo del estrés y .75 en Intrapersonal; obteniéndose valores similares a los hallados por los autores de la prueba en la validación de la misma en una muestra de un contexto similar al de la investigación que presentamos.

\section{Procedimiento}

Los dos instrumentos empleados se aplicaron de forma colectiva a los participantes en sus aulas de clase. Se pidió su colaboración para el estudio sin ningún tipo de coacción. Antes de que contestaran los instrumentos, se avisó de que se respetaría el anonimato de las respuestas y la confidencialidad de los resultados. Los cuestionarios fueron auto-administrados pero se explicaron las normas de aplicación de los mismos por miembros del equipo de investigación, quienes estuvieron presentes mientras eran cumplimentados para resolver dudas y verificar la correcta contestación, siendo la duración media del proceso de 20 minutos.

\section{Análisis de los datos}

Para el análisis de los datos se empleó el programa estadístico IBM SPSS Statistics versión 22 (SPSS Inc., Chicago, USA). Se ha realizado un análisis descriptivo, para conocer la existencia de diferencias significativas a nivel estadístico en función del género, edad, grado y curso/nivel, se utilizó la prueba $t$ de Student de diferencias de medias para muestras independientes. Se verificaron los supuestos de independencia de los datos, normalidad y homocedasticidad (Gil-Pascual, 2015), y se informa del tamaño del efecto. Se emplea las prueba paramétrica descrita al sostenerse la asunción de normalidad en todos los casos, no siendo necesario el cálculo de pruebas no paramétricas. Finalmente, se realiza un estudio de regresión lineal mediante el método de pasos sucesivos para predecir qué dimensiones o factores explican en mayor medida la IE global. En todos los análisis se ha empleado un nivel confianza del 95\% (significación $p<.05)$.

\section{Resultados}

\section{Valoraciones de la IE y correlaciones entre los factores del EQ-i Short Form}

En primer lugar, para dar respuesta a los dos primeros objetivos de la investigación, vamos a presentar los resultados obtenidos para el total de la muestra $(n=460)$ en cada uno de los factores del instrumento empleado (EQi-Short Form) y las correlaciones entre cada uno de ellos, así como de la fiabilidad de las puntuaciones de cada factor (véase Tabla 1). 
Tabla 1. Correlaciones ( $r$ ) entre los factores, descriptivos y fiabilidad de las puntuaciones

\begin{tabular}{ccccc}
\hline & $\begin{array}{c}\text { INTER } \\
{[\text { Rango 7-35] }}\end{array}$ & $\begin{array}{c}\text { ADAPT } \\
{[\text { Rango 5-25] }}\end{array}$ & $\begin{array}{c}\text { M-EST } \\
{[\text { Rango 8-40] }}\end{array}$ & $\begin{array}{c}\text { INTRA } \\
{[\text { Rango 8-40] }}\end{array}$ \\
\hline INTER & - & & & \\
ADAPT & $.265^{* *}$ & - & & \\
M-EST & .034 & $.143^{* *}$ & - & - \\
INTRA & $.095^{*}$ & $.214^{* *}$ & $.495^{* *}$ & \\
M $(D T)$ & $29.53( \pm 3.42)$ & $18.53( \pm 3.19)$ & $27.13( \pm 7.06)$ & $30.16( \pm 5.34)$ \\
Alpha $(\alpha)$ & .67 & .74 & .88 & .75 \\
\hline
\end{tabular}

Nota:

(1) INTER = Interpersonal, ADAPT = Adaptabilidad, M-EST = Manejo del Estrés, INTRA = Intrapersonal.

(2) ${ }^{*}=p<.05 ;{ }^{* *}=p<.01$.

(3) $M=$ Media, $D T=$ Desviación Típica, $\alpha=$ Fiabilidad de las puntuaciones (consistencia interna).

Las correlaciones entre los factores son positivas y significativas en todos los casos $(p<.05$ ó $p<.01)$ aunque de moderada magnitud (valores de $r_{460}$ comprendidos entre .10 y .50), salvo entre los factores Interpersonal y Manejo del Estrés donde no hay significatividad $\left(r_{460}=.034, p>.05 \mathrm{~ns}\right)$. Las puntuaciones medias en cada dimensión son elevadas, superan con creces de manera global, las valoraciones medias en cada una en función del rango de puntuaciones posibles. Asimismo, tal y como hemos presentado en el método, la fiabilidad de las puntuaciones del instrumento presentan valores aceptables de consistencia interna comprendidas entre 0.67 y .88.

\section{Diferencias en función de las variables sociodemográficas}

Para analizar las diferencias en función de las variables sociodemográficas consideradas: género (hombre vs. mujer), edad ( $<25$ años vs. $\geq 25$ años), grado (Infantil vs. Primaria) y curso/nivel $\left(3^{\circ}\right.$ vs. $\left.4^{\circ}\right)$; hemos realizado diversas pruebas $t$ Student (diferencias de medias) para muestras independientes (véase Tabla 2).

En función del género, los resultados indican que existen diferencias significativas en los factores Interpersonal $\left(t_{458}=-6.956, p=.000, d=-.73\right)$, a favor de las mujeres con un valor del tamaño del efecto $(d=-.73)$ cercano a la consideración de efecto grande siguiendo los criterios clásicos de Cohen (1988); en Manejo del Estrés la diferencia es a favor de los hombres $\left(t_{458}=2.308, p=.021\right.$, Efecto $\left.d=.25\right)$, siendo el único factor en el que los hombres obtienen valoraciones medias superiores a la mujeres de la muestra. Al analizar las diferencias en función de la edad, se ha encontrado significatividad en Adaptabilidad $\left(t_{458}=-4.455, p=.000\right)$ y en Intrapersonal $\left(t_{458}=-2.319, p=.021\right)$, en ambos casos con valores del tamaño del efecto medianos $(d=-.63$ y $d=-.32$, respectivamente), siendo las diferencias favorables a los participantes de mayor edad ( $\geq 25$ años) en todos los casos.

Las puntuaciones obtenidas en la variable grado (Infantil vs. Primaria), informan de diferencias significativas en todos los factores salvo en Adaptabilidad. Las evidencias aparecen por tanto en los factores Interpersonal $\left(t_{458}=5.303, p=.000, d=.49\right)$, Manejo del Estrés $\left(t_{458}=2.615, p=.009, d=.24\right)$ e Intrapersonal $\left(t_{458}=2.744, p=.006\right.$, 
$d=.25)$, a favor del alumnado de Educación Infantil en todos ellos, siendo el efecto de tamaño mediano. Por último, en cuanto al curso/nivel $\left(3^{\circ} \mathrm{vs} .4^{\circ}\right)$ el comportamiento de los resultados es similar a la variable grado, mostrándose diferencias significativas en los mismos factores que en ésta, Interpersonal $\left(t_{458}=-2.735 p=.006, d=-.25\right)$, Manejo del Estrés $\left(t_{458}=-1.975 p=.049, d=-.18\right)$ e Intrapersonal $\left(t_{458}=-2.612 p=.009, d=-.24\right)$, con valores medios más altos en los estudiantes de $4^{\circ}$ curso/nivel, siendo el efecto pequeño en todos los casos.

Tabla 2. Diferencias de medias en función de las variables sociodemográficas (t de Student)

\begin{tabular}{|c|c|c|c|c|c|}
\hline Género & $\begin{array}{c}\text { Hombres }(n=104) \\
M(D T)\end{array}$ & $\begin{array}{c}\text { Mujeres }(n=356) \\
M(D T)\end{array}$ & $t_{(458)}$ & $p$ & Efecto $(d)$ \\
\hline INTER (Rango 7-35) & $27.57( \pm 3.81)$ & $30.10( \pm 3.08)$ & -6.956 & $.000^{* *}$ & -.73 \\
\hline ADAPT (Rango 5-25) & $18.43( \pm 3.32)$ & $18.56( \pm 3.16)$ & -378 & .706 & -.04 \\
\hline M-EST (Rango 8-40) & $28.53( \pm 7.19)$ & $26.73( \pm 6.98)$ & 2.308 & $.021^{*}$ & .25 \\
\hline INTRA (Rango 8-40) & $29.94( \pm 5.06)$ & $30.23( \pm 5.42)$ & -.492 & .623 & -.05 \\
\hline Edad & $\begin{array}{c}<25 \text { años }(n=404) \\
M(D T)\end{array}$ & $\begin{array}{c}\geq 25 \text { años }(n=56) \\
M(D T)\end{array}$ & $t_{(458)}$ & $p$ & Efecto $(d)$ \\
\hline INTER (Rango 7-35) & $29.44( \pm 3.47)$ & $30.17( \pm 2.99)$ & -1.502 & .134 & -.22 \\
\hline ADAPT (Rango 5-25) & $18.29( \pm 3.12)$ & $20.28( \pm 3.16)$ & -4.455 & $.000^{* *}$ & -.63 \\
\hline M-EST (Rango 8-40) & $26.97( \pm 6.80)$ & $28.33( \pm 8.66)$ & -1.132 & .262 & -.17 \\
\hline INTRA (Rango 8-40) & $29.95( \pm 5.28)$ & $31.71( \pm 5.58)$ & -2.319 & $.021^{*}$ & -.32 \\
\hline Grado & $\begin{array}{c}\text { Infantil }(n=235) \\
M(D T)\end{array}$ & $\begin{array}{c}\text { Primaria }(n=225) \\
M(D T)\end{array}$ & $t_{(458)}$ & $p$ & Efecto $(d)$ \\
\hline INTER (Rango 7-35) & $30.34( \pm 3.11)$ & $28.69( \pm 3.53)$ & 5.303 & $.000^{* *}$ & .49 \\
\hline ADAPT (Rango 5-25) & $18.42( \pm 3.31)$ & $18.65( \pm 3.07)$ & -.793 & .428 & -.07 \\
\hline M-EST (Rango 8-40) & $27.97( \pm 6.54)$ & $26.26( \pm 7.47)$ & 2.615 & $.009^{*}$ & .24 \\
\hline INTRA (Rango 8-40) & $30.83( \pm 5.24)$ & $29.47( \pm 5.37)$ & 2.744 & $.006^{*}$ & .25 \\
\hline Curso & $\begin{array}{l}\text { Tercero }(n=200) \\
M(D T)\end{array}$ & $\begin{array}{c}\text { Cuarto }(n=260) \\
M(D T)\end{array}$ & $t_{(458)}$ & $p$ & Efecto $(d)$ \\
\hline INTER (Rango 7-35) & $29.04( \pm 3.20)$ & $29.91( \pm 3.54)$ & -2.735 & $.006^{*}$ & -.25 \\
\hline ADAPT (Rango 5-25) & $18.66( \pm 2.97)$ & $18.44( \pm 3.36)$ & .723 & .470 & .06 \\
\hline M-EST (Rango 8-40) & $26.40( \pm 7.12)$ & $27.70( \pm 6.97)$ & -1.975 & $.049^{*}$ & -.18 \\
\hline INTRA (Rango 8-40) & $29.43( \pm 5.37)$ & $30.73( \pm 5.26)$ & -2.612 & $.009^{*}$ & -.24 \\
\hline
\end{tabular}

Nota:

(1) INTER = Interpersonal, ADAPT $=$ Adaptabilidad, M-EST = Manejo del Estrés, INTRA = Intrapersonal.

(2) ${ }^{*}=p<.05 ; * *=p<.01 . M=$ Media, $D T=$ Desviación Típica.

(3) El tamaño del efecto estadístico está expresado con el valor $d$ de Cohen (1988): pequeño=.2, mediano $=.5$ y grande $=.8$. 


\section{Estudio de regresión}

Para analizar qué variables son las que más predicen la IE, hemos realizado una regresión lineal usando el método de pasos sucesivos (Véase Tabla 3), considerado como variables predictoras cada uno de los factores del EQ-i Short Form y como variable criterio la valoración global de la IE.

Tabla 3. Análisis de regresión lineal (pasos sucesivos), variable criterio: Inteligencia Emocional

\begin{tabular}{clccc}
\hline Modelo & Variables predictoras & Beta & $t$ & $p$ \\
\hline \multirow{2}{*}{ Modelo 1 } & (Constante) & & 47.113 & $.000^{* *}$ \\
& Manejo del Estrés (M-EST) & .756 & 24.736 & $.000^{* *}$ \\
\hline \multirow{2}{*}{ Modelo 2 } & (Constante) & .685 & 23.255 & $.000^{* *}$ \\
& Manejo del Estrés (M-EST) & .496 & 24.233 & $.000^{* *}$ \\
& Adaptabilidad (ADAPT) & & 20.458 & $.000^{* *}$ \\
\hline \multirow{2}{*}{ Modelo 3 } & (Constante) & .499 & 33.973 & $.000^{* *}$ \\
& Manejo del Estrés (M-EST) & .438 & 33.558 & $.000^{* *}$ \\
& Adaptabilidad (ADAPT) & .393 & 26.397 & $.000^{* *}$ \\
\hline \multirow{5}{*}{ Modelo 4 } & Antrapersonal (INTRA) & & .157 & .875 \\
& (Constante) & .508 & 324.330 & $.000^{* *}$ \\
& Manejo del Estrés (M-EST) & .366 & 254.606 & $.000^{* *}$ \\
& Intrapersonal (INTRA) & .377 & 237.784 & $.000^{* *}$ \\
& Interpersonal (INTER) & .281 & 199.368 & $.000^{* *}$ \\
\hline
\end{tabular}

Nota:

(1) INTER = Interpersonal, ADAPT = Adaptabilidad, M-EST = Manejo del Estrés, INTRA = Intrapersonal.

(2) $* *=p<.01$.

El resumen del análisis indica que se han incluido en el modelo 4 todos los factores del instrumento: Manejo del Estrés (M-EST), Adaptabilidad (ADAPT), Intrapersonal (INTRA) e Interpersonal (INTER), siendo todos predictores positivos de la IE. El mayor poder apreciado en los coeficientes tipificados lo tiene M-EST (Beta=.508; $p<.01)$, seguido de la ADAPT $($ Beta $=.366 ; p<.01)$, INTRA (Beta=.237; $p<.01)$ y el último lugar INTER $($ Beta $=.281 ; p<.01)$, existiendo en todos ellos un valor de $t$ significativo $(p<.01)$.

\section{DisCUSIÓN Y CONCLUSIONES}

Las valoraciones de la IE recogidas a través del EQ-i Short Form han sido elevadas en los sujetos de la muestra y las correlaciones entre los distintos factores del instrumento son significativas en todos los casos (tan solo no hay significatividad entre los factores Interpersonal y el Manejo del Estrés, aunque si hay relación directa pero no significativa). Estos resultados son concordantes con los obtenidos por otros autores empleando diversas versiones del mismo instrumento (Di Fabio y Sklofske, 2014; Di Fabio, Sklofske y Tremblay, 2016; Furhham, 2016). 
En relación con las variables sociodemográficas, en el género se aprecia significatividad en las diferencias en dos factores, coincidiendo con estudios que analizan la IE rasgo en distintos países europeos como Reino Unido (Furham, 2016; Petrides, 2009), Italia (Di Fabio, Sklofske y Tremblay, 2016), Francia (Mikolajczak et al., 2007), Grecia (Stamatopoulou, Galanis y Prezerakos, 2016), Serbia (Marjanović y Dimitrijević, 2014) o en España (Fortes-Vilaltella et al., 2013). La existencia de diferencias de género en la IE medida con autoinformes es un aspecto contradictorio al no estar presentes en todos los estudios que emplean instrumentos similares (Sala-Roca, 2002), las inconsistencias en los resultados pueden estar condicionadas por los instrumentos usados y los modelos teóricos en los que se basan (Gartzia, Aritzeta, Balluerka y Barberá, 2012; Gartzia y López-Zafra, 2014) tal y como queda de manifiesto en el amplio meta-análisis realizado por Joseph y Newman (2010).

Para la variable edad hay evidencias significativas algunos factores, siendo favorables a los estudiantes de mayor edad, datos que siguen la misma tendencia que los obtenidos en otros trabajos (Mikolajczak et al., 2007; Stamatopoulou, Galanis y Prezerakos, 2016; Yeung, Wong y Lok, 2011); al igual que ocurre en relación al nivel educativo (Manoharan, 2008; Stamatopoulou, Galanis y Prezerakos, 2016) siendo las diferencias favorables a los de nivel superior. Finalmente, para la variable grado (Infantil vs. Primaria), todos los factores menos uno (Adaptabilidad) informan de diferencias significativas, siendo favorables las mismas a los estudiantes de Educación Infantil (en los tres casos), las cuales son en su gran mayoría mujeres, algo que puede condicionar que las diferencias sean significativas, ya que es preciso recordar que las mujeres de nuestra muestra, de manera global, obtenían puntuaciones más elevadas que los hombres. En otros estudios realizados con universitarios españoles de diversas titulaciones (Sala-Roca, 2002) se han encontrado diferencias significativas en IE a favor de los estudiantes de magisterio con respecto a otros títulos de educación, por lo que parece quedar de manifiesto la mayor sensibilidad de esta titulación por los aspectos emocionales. Encontrar estudios con los que comparar los resultados de la variable grado en el panorama internacional es muy complicado, ya que la denominación de los mismos cambia en cada país.

El estudio de regresión informa que el factor que más predice la IE rasgo es el Manejo del Estrés, seguido de la Adaptabilidad, Intrapersonal e Interpersonal. Examinar el constructo IE y analizar el peso de la predicción de la misma por sus factores es una tarea compleja, ya que no solo se tiene que contemplar qué modelo se sigue (modelo IE habilidad o modelo IE rasgo), sino que además los resultados pueden diferir en función del instrumento que empleemos (Ferrándiz et al., 2012). En próximos trabajos sería de utilidad contemplar más de un instrumento para evaluar la IE rasgo y contrastar los resultados.

\section{Conclusiones}

Es importante considerar que la adquisición de conocimientos meramente académicos no es suficiente para conseguir el éxito escolar debiéndose valorar las implicaciones educativas de los aspectos emocionales en las aulas, en especial en lo relacionado con el bienestar emocional (Antoñanzas et al., 2014) y sus repercusiones en la mejora de convivencia escolar (Sáez, Lavega, Mateu y Rovira, 2014). El desarrollo de las competencias emocionales es clave para el logro del bienestar, por este motivo es necesario que la educación de las emociones se contemple en los currícula educativos 
(Bisquerra, Pérez-González y García-Navarro, 2015), observando la relación existente entre los estilos educativos y el desarrollo emocional, algo que puede favorece el bienestar tanto del alumnado como del profesorado (Sala-Roca y Marzo-Ruiz, 2013).

Los formadores de los futuros docentes deben procurar que las creencias de los estudiantes sobre las emociones sean la adecuadas, de lo contrario, estos futuros docentes tenderán a reproducir modelos ausentes de ellas (Gairín, Murillo, Escolano, Roncal y Jiménez, 2013). En esta línea hay que destacar la importancia de estudios que realicen propuestas de intervención para el desarrollo de la IE, en especial durante la educación infantil y primaria (Pérez-Escoda, Filella-Guiu, Soldevilla-Benet y Fondevila, 2013).

Coincidimos con Abarca-Castillo, Marzo-Ruiz y Sala-Roca (2002) en que la práctica educativa diaria puede potenciar el desarrollo emocional del alumnado con tan solo realizar pequeñas modificaciones metodológicas de las clases y las maneras de interacción entre los escolares. El profesorado está muy sensibilizado ante la necesidad de incluir la educación emocional pero que no ha recibido la formación necesaria, por lo que trabajar la educación emocional está al alcance de todo el profesorado, con el desarrollo de pequeños esfuerzos de la administración educativa.

Pero nuestro estudio no puede finalizar sin reflexionar sobre sus limitaciones y la prospectiva del mismo. En este sentido es preciso valorar los sesgos que pueden aparecer en los estudios que emplean pruebas de autoinforme, por el efecto de la deseabilidad social, sin duda en futuros trabajos emplear además de autoinformes otro tipo de medidas (medidas de ejecución e informes de evaluación $360^{\circ}$ por medio de observadores externos) podrían servir de ayuda para complementar las evidencias obtenidas.

A pesar de la dilatada producción en torno a la IE, ésta es una línea de investigación joven aún, por lo que habrá que seguir trabajando en ella realizando trabajos con muestras más amplias, de tipo transcultural y con estudios longitudinales, algo que favorecería extraer conclusiones más sólidas sobre de la utilidad y la necesidad de las emociones. Es preciso actuar con mucha cautela en la generalización de los resultados a otros contextos diferentes al nuestro, en este sentido, para futuros trabajos, contemplamos la necesidad de incluir participantes de otras universidades.

La imagen que los docentes tienen sobre el contexto de enseñanza, condiciona su forma de enseñar, su manera de estructurar la misma y su implicación con los estudiantes (Hernández-Pina, Maquilón-Sánchez y Monroy-Hernández, 2012).

Finalmente, aunque no sea responsabilidad directa nuestra, la información que se ofrece en nuestro estudio podrá ser de utilidad para las personas que ocupen puestos de responsabilidad en la gobernanza de las universidades, facultades, centros, departamentos, etc.; en especial en aquellas áreas relacionadas directamente con la formación inicial del profesorado, para que tomen las oportunas decisiones en la planificación, desarrollo y evaluación de los estudios de los que sean responsables.

\section{AgradeCimientos}

Agradecemos a la Dra. Esther López-Zafra, al Dr. Manuel Pulido-Martos y a la Dra. Pilar Berrios-Martos, autores de la adaptación al español del EQ-i Short Form (López-Zafra et al., 2014), la ayuda prestada al autorizarnos a emplear el instrumento y facilitarnos las normas de la corrección del mismo. 


\section{REFERENCIAS BIBLIOGRÁFICAS}

Abarca-Castillo, M., Marzo-Ruiz, L. y Sala-Roca, J. (2002). La educación emocional en la práctica educativa de Primaria. Bordón, Revista de Pedagogía, 54(4), 505-518.

Adina, A. y Clipa, O. (2012). Teachers' satisfaction with life, job satisfaction and their emotional intelligence. Procedia - Social and Behavioral Sciences, 33, 498-502. DOI: http://doi.org/10.1016/j.sbspro.2012.01.171.

Andrei, F. y Petrides, K. V. (2013). Trait emotional intelligence and somatic complaints with reference to positive and negative mood. Psihologija, 46(1), 5-15. DOI: http:// doi.org/10.2298/PSI1301005A.

Andrei, F., Smith, M. M., Surcinelli, P., Baldaro, B. y Saklofske, D. (2015). The trait emotional intelligence questionnaire: Internal structure, criterion and incremental validity in an Italian sample. Measurement and Evaluation in Counseling and Development, 49(1), 34-45. DOI: http://doi.org/10.1177/0748175615596786.

Antoñanzas, J. L., Salavera, C., Teruel, P., Sisamón, C., Ginto, A., Anaya, A. y BarceIona, D. (2014). Emotional intelligence and personality in student teachers. Procedia, Social and Behavioral Sciences, 132, 492-496. DOI: http://doi.org/10.1016/j. sbspro.2014.04.342.

Ato, M., López, J. J. y Benavente, A. (2013). Un sistema de clasificación de los diseños de investigación en psicología. Anales de Psicología, 29(3), 1038-1059. DOI: http://doi.org/10.6018/analesps.29.3.178511.

Bar-On, R. (1997). The emotional intelligence inventory (EQ-i): Technical manual. Toronto: Multi-Health Systems.

Bar-On, R. (2002). Bar-On Emotional Quotient Short Form (EQ-I: Short): Technical manual. Toronto: Multi-Health Systems.

Bar-On, R. (2006). The Bar-On model of Emotional-Social Intelligence (ESI). Psicothema, 18, 13-25.

Bisquerra, R., Pérez-González, J. C. y García-Navarro, E. (2015). Inteligencia emocional en educación. Madrid: Síntesis.

Cazalla-Luna, N. y Molero, D. (2014). Inteligencia Emocional Percibida, Ansiedad y Afectos en estudiantes universitarios. Revista Española de Orientación y Psicopedagogía, 25(3), 56-73. DOI: http://doi.org/10.5944/reop.vol.25.num.3.2014.13858.

Cazalla-Luna, N. y Molero, D. (2016). Inteligencia emocional percibida, disposición al optimismo-pesimismo, satisfacción vital y personalidad de docentes en su formación inicial. Revista de Investigación Educativa, 34(1), 241-258. DOI: http:// doi.org/10.6018/rie.34.1.220701.

Cohen, J. (1988). Statistical power analysis for the behavioral sciencies. Hillsdale, New York: Erlbaum.

Di Fabio, A. y Saklofske, D. (2014). Promoting individual resources: The challenge of trail emotional intelligence. Personality and Individual Differences, 65, 19-23. DOI: http://doi.org/10.1016/j.paid.2014.01-026.

Di Fabio, A., Saklofske, D. y Tremblay, P. F. (2016). Psychometric properties of the Italian trail emotional intelligence. Personality and Individual Differences, 96, 198201. DOI: http://doi.org/10.1016/j.paid.2016.03-009.

Extremera, N., Fernández-Berrocal, P., Mestre, J. y Guil, R. (2004). Medidas de evaluación de la Inteligencia emocional. Revista Latinoamericana de Psicología, 36(2), 209-228. 
Ferrándiz, C., Hernández, D., Bermejo, R., Ferrando, M. y Sáinz, M. (2012). Social and emotional intelligence in childhood and adolescence: Spanish validation of a measurement instrument. Revista de Psicodidáctica, 17(2), 309-338.

Fortes-Vilaltella, M., Oriol, X., Filella-Guiu, G., Del Arco, I. y Soldevila, A. (2013). Inteligencia emocional y personalidad en las diferentes áreas de conocimiento de los estudiantes universitarios. Revista Electrónica Interuniversitaria de Formación del Profesorado, 16(1), 109-120. DOI: http://doi.org/10.6018/reifop.16.1.179471.

Freudenthaler, H. H., Neubauer, C. A., Gabler, P., Scherl, W. G. y Rindermann, H. (2008). Testing and validating the trait emotional intelligence questionnaire (TEIQue) in a German-speaking simple. Personality and Individual Differences, 45(7), 673-678. DOI: http://doi.org/10.1016/j.paid.2008.07.014.

Furham, A. (2016). The Relationship between Cognitve Ability, Emotional Intelligence and Creativity. Psychology, 7, 193-197. DOI: http://doi.org/10.4236/psych.2016.72021.

Furnham, A. y Christoforou, I. (2007). Personality traits, emotional intelligence, andmultiple happiness. North American Journal of Psychology, 9(3), 439-462.

Gairín-Sallán, J., Murillo-Ramón, J., Escolano-Vizcarra, R., Roncal-Gómez, L. y Jiménez-Gestal, C. (2013). Perfil emocional y competencias matemáticas de los estudiantes del grado de educación primaria. Contextos Educativos. Revista de Educación, 15, 107-134. DOI: http://doi.org/10.18172/con.658.

Gartzia, L. y López-Zafra, E. (2014). Perceptions of Gender Differences in Self-Report Measures of Emotional Intelligence. Sex Roles, 70, 479-495. DOI: http://doi.org/ 10.1007/s/11199-014-0368-6.

Gartzia, L., Aritzeta, A., Balluerka, N. y Barberá, E. (2012). Inteligencia emocional y género: más allá de las diferencias sexuales. Anales de Psicología, 28(2), 567-575. DOI: http://doi.org/10.6018/analesps.28.2124111.

Gil-Pascual, J. A. (2015). Análisis estadísticos básicos. Cuándo y cuáles emplear. En A. Pantoja (coord.), Manual básico para la realización de tesinas, tesis y trabajos de investigación (pp. 342-359). Madrid: EOS Universitaria.

Hernández-Pina, F., Maquilón-Sánchez, J. y Monroy-Hernández, F. (2012). Estudio de los enfoques de enseñanza en profesorado de Educación Primaria. Profesorado, Revista de Currículum y Formación del profesorado, 16(1), 61-77.

Jacobs, I., Sim, C. W. y Zimmermann, J. (2015). The German TEIQue-SF: Factorial structure and relations to agentic and communal traits and mental health. Personality and Individual Differences, 72, 189-194. DOI: http://doi.org/10.1016/j.paid.2014.09. 003.

Jiménez, M. ${ }^{\text {a }}$. y López-Zafra, E. (2009). Inteligencia emocional y rendimiento escolar: estado actual de la cuestión. Revista Latinoamericana de Psicología, 41(1), 69-79.

Joseph, D. L. y Newman, D. A. (2010). Emotional intelligence: An integrative metaanalysis and cascading model. Journal of Applied Psychology, 95(1), 54-78. DOI: http://doi.org/10.1037/a0017286.

Kong, F., Zhao, J. y You, X. (2012). Emotional intelligence and life satisfaction in Chinese university students: The mediating role of self-esteem and social support. Personality and Individual Differences, 53(8), 1039-1043. DOI: http://doi. org/10.1016/j.paid.2012.07.032. 
López-Zafra, E., Pulido-Martos, M. y Berrios-Martos, P. (2014). EQ-i Versión Corta (EQi-C). Adaptación y validación al español del EQ-i en universitarios. Boletín de Psicología, 110, 21-36.

López-Zafra, E., Pulido-Martos, M., Berrios, M. P. y Augusto-Landa, J. M. (2012). Psychometric properties of the Spanish version of the Work Group Emotional Intelligence Profile-Short version. Psicothema, 24, 495-502.

Manoharan, P. K. (2008). Education and personality development. New Delphi: APH Publishing Corporation.

Marjanović, Z. J. y Dimitrijević, A. A. (2014). Reliability, construct and criterion-related validity of the Serbian adaptation of the Trait Emotional Intelligence Questionnaire (TEIQue). Psihologija, 47(2), 249-262. DOl: http://doi.org/10.2298/ PSI1402249J.

Mayer, J. D. y Salovey, P. (1997). What is emotional intelligence? En P. Salovey y D. Sluyter (eds.), Emotional Development and Emotional Intelligence: implications for educators. New York: Basic Books, pp. 3-31.

Mayer, J. D., Roberts, R. D. y Barsade, S. G. (2008). Human abilities: Emotional InteIligence. Annual review of Psychology, 59, 507-536.

Mikolajczak, M., Luminet, O., Leroy, C. y Roy, E. (2007). Psychometric properties of the Trait Emotional Intelligence Questionnaire: Factor structure, reliability, construct, and incremental validity in a French-speaking population. Journal of Personality Assessment, 88(3), 338-353. DOI: http://doi.org/10.1080/00223890701333431.

Mikolajczak, M., Menil, C. y Luminet, O. (2007). Explaining the protective effect of trait emotional intelligence regarding occupational stress: Exploration of emotional labour processes. Journal of Research in Personality, 41(5), 1107-1117. DOI: http://doi.org/10. 1016/j.jrp.2007.01.003.

Parker, J. D. A., Keefer, K. V. y Wood, L. M. (2011). Toward a brief multidimensional assessment of emotional intelligence: Psychometric properties of the Emotional Quotient Inventory-Short Form. Psychological Assessment, 23, 762-777. DOI: http://doi.org/10.1037/a0023289.

Pérez-Escoda, N., Filella-Guiu, G., Soldevila-Benet, A. y Fondevila, A. (2013). Evaluación de un programa de educación emocional para profesorado de primaria. Educación XX1, 16(1), 233-254. DOl: http://doi.org/10.5944/educxx1.16.1.725.

Petrides, K. V. (2009). Psychometric properties of the Trait Emotional Intelligence Questionnaire. En C. Stough, D. H. Saklofske y J. D. Parker (eds.), Advances in the assessment of emotional intelligence. New York: Springer. DOI: http://doi. org/10.1007/978-0-387-88370-0_5.

Petrides, K. V. y Furnham, A. (2000). On the dimensional structure of emotional intelligence. Personality and Individual Differences, 29, 313-320. DOI: http://doi. org/10.1016/S0191-8869(99)00195-6.

Petrides, K. V. y Furnham, A. (2001). Trait emotional intelligence: Psychometric investigation with reference to established trait taxonomies. European Journal of Personality, 15, 425-428. DOI: http://doi.org/10.1002/per.416.

Rey, L. y Extremera, N. (2012). Inteligencia emocional percibida, felicidad y estrategias distractoras en adolescentes. Boletín de Psicología, 104, 87-101. 
Roberts, R. D., MacCann, C., Matthews, G. y Zeidner, M. (2010). Emotional InteIligence: Toward a Consensus of Models and Measures. Social and Personality Psychology Compass, 4(10), 821-840. DOl: http://doi.org/10.1111/j.17519004.2010.00277.x.

Sáez, U., Lavega, P., Mateu, M. y Rovira, G. (2014). Emociones positivas y educación de la convivencia escolar. Contribución de la expresión motriz cooperativa. Revista de Investigación Educativa, 32(2), 309-326. DOI: http://doi.org/10.6018/ rie.32.2.183911.

Sáinz, M., Ferrándiz, C., Fernández, C. y Ferrando, M. (2014). Propiedades psicométricas del Inventario de Cociente Emocional EQ-i: YV en alumnos superdotados y talentosos. Revista de Investigación Educativa, 32(1), 41-55. DOI: http://doi. org/10.6018/rie.32.1.162501.

Sala-Roca, J. (2002). Ideas previas sobre la docencia y competencias emocionales en estudiantes de ciencias de la educación. Revista Española de Pedagogía, 60(223), 543-557.

Sala-Roca, J. y Marzo-Ruiz, L. (2013). Emociones y procesos educativos: los estilos educativos y las emociones. En P. Darder-Vidal (coord.), Aprender y educar con bienestar y empatía. La formación emocional del profesorado. Barcelona: Octaedro. pp. 103-122.

Salovey, P. y Grewal, D. (2005). The Science of emotional intelligence. Currents Directions in Psychological Science, 14, 281-285. DOI: http://doi.org/10.1111/ j.0963-7214.2005.00381.x.

Salovey, P. y Mayer, J. D. (1990). Emotional Intelligence. Imagination, Cognition and Personality, 9, 185-211. DOI: http://doi.org/10.2190/DUGG-P24E-52WK-6CDG.

Stamatopoulou, M., Galanis, P. y Prezerakos, P. (2016). Psychometric propertites of the Greek traslation of the Trail Emotional Intelligence Questionnaire-Short Form. Personality and Individual Differences, 95, 80-84. DOI: http://doi.org/10.1016/j. paid.2016.02-035.

Swami, V., Begum, S. y Petrides, K. V. (2010). Associations between trait emotional intelligence, actual-ideal weight discrepancy, and positive body image. Personality and Individual Differences, 49(5), 485-489. DOI: http://doi.org/10.1016/j. paid.2010.05.009.

Valadez-Sierra, M. D., Borges del Rosal, M. A., Ruvalcaba-Romero, N., Villegas, K. y Lorenzo, M. (2013). Emotional Intelligence and its Relationship with Gender, Academic Performance and Intellectual Abilities of Undergraduates. Electronic Journal of Research in Educational Psychology, 11(2), 395-412. DOI: http://doi. org/10.14204/ejrep.30.12204.

Weaving, J., Orgeta, V., Orrell, M. y Petrides, K. V. (2014). Predicting anxiety in carers of people with dementia: The role of trait emotional intelligence. International Psychogeriatrics, 26(7), 1201-1209. DOI: http://doi.org/10.1017/ S1041610214000404.

Yeung, D. Y.,Wong, C. K. y Lok, D. P. (2011). Emotion regulation mediates age differences in emotions. Aging \& Mental Health, 15(3), 414-418. DOI: http://doi. org/10.1080/ 13607863.2010.536136. 